\title{
A Task-Type-Based Algorithm for the Energy-Aware Profit Maximizing Scheduling Problem in Heterogeneous computing systems
}

\author{
Weidong Li, Xi Liu, Xuejie Zhang, Xiaobo Cai \\ Yunnan University \\ Kunming, China \\ Email: \{weidong, xliu,xjzhang, xbcai\}@ynu.edu.cn
}

\begin{abstract}
In this paper, we design an efficient algorithm for the energy-aware profit maximizing scheduling problem, where the high performance computing system administrator is to maximize the profit per unit time. The running time of the proposed algorithm is depending on the number of task types, while the running time of the previous algorithm is depending on the number of tasks. Moreover, we prove that the worst-case performance ratio is close to 2 , which maybe the best result. Simulation experiments show that the proposed algorithm is more accurate than the previous method.
\end{abstract}

Keywords-high performance computing; scheduling; resource allocation; approximation algorithm; bag-of-tasks

\section{INTRODUCTION}

\section{A. Background and Motivation}

In high-performance computing (HPC) systems, it is well known that when the performance is increased, the power consumption is increased, as well as the electricity costs for the operators are increased. Recently, the high cost of the HPC systems has lead to research that designs an efficient resource allocation algorithm to reduce the required energy consumption [1]. By combining the energy and performance objectives into a single profit objective, Tarplee et al. [1] introduced a novel monetary-based model for HPC where there is a financial distinction between the service provider and the users. In HPC systems, there are two important facts: (a) The HPC systems are often composed of different types of machines; (b) There are a large number of tasks but only small number of task types. By solving a linear program and rounding carefully, they [1] designed an efficient algorithm to find a feasible schedule.

In [1], a lower bound on the finishing times of a machine type is used to replace makespan, which is defined as the maximum finishing time of all machines. Therefore, the proposed mathematical model is inaccurate. For the proposed algorithm [1], in the rounding process, the energy consumption maybe increased, which can be avoided by using a different method. Moreover, the running time is depending on the number of tasks, which can be improved, too. Most importantly, the worst-case performance ratio of the proposed algorithm [1] is not given.

\section{B. Contributions and Outline}

This paper presents an accurate mathematical model for the problem proposed in [1]. A polynomial-time algorithm is then developed to find a feasible solution for the proposed model.

The contributions of this paper are:

1) An accurate mathematical model;

2) A task-type-based algorithm to find a more accurate feasible solution, whose running time is independent of the number of tasks;

3) The worst-case performance ratio.

The remainder of this paper is organized as follows. The next section proposes the accurate mathematical model. Section III presents the task-type-based algorithm and proves the worst-case performance ratio. Section IV gives the experimental results. The last section discusses the useful extensions to the proposed model and lists ideas for future work.

\section{The Mathematical Model}

As in [1], a user submits a bag-of-tasks to process, where each task is indivisible and independent of all the other tasks. The cost to the organization for processing a bag-of-tasks is the cost of electricity. The organization or service provider should maximum the profit per bag, which is equal to the price minus the cost. However, the bag-of-tasks can take a considerable amount of time to compute when trying to increase the profit by reducing electricity costs. Thus, it is more reasonable for an organization to maximize the profit per unit time.

Formally, assume that there are $T$ task types and $M$ machine types. Let $\mathcal{T}_{i}$ be the set of tasks of type $i$ and $T_{i}$ be the number of tasks in $\mathcal{T}_{i}$. Similarly, let $\mathcal{M}_{j}$ be the set of machines of type $j$ and $M_{j}$ be the number of machines in $\mathcal{M}_{j}$. Denote by $x_{i j}$ the number of tasks of type $i$ assigned to a machine of type $j$, where $x_{i j}$ is the primary decision variable in the optimization problem. As the definitions frequently used in scheduling algorithms [1], let ETC be a $T \times M$ matrix where $E T C_{i j}$ is the estimated time to compute for a task $i$ on a machine $j$. Similarly, let APC be a $T \times M$ matrix where $A P C_{i j}$ is the average power consumption for a task $i$ on a machine $j$. 
Since tasks are indivisible in most cases, the $x_{i j}$ tasks of type $i$ may not be allocated equally to the $M_{j}$ machines of type $j$. For every machine $j_{k} \in \mathcal{M}_{j}$, let $x_{i j k}$ be the number of tasks of type $i$ assigned to machine $j_{k}$. Clearly, $x_{i j}=$ $\sum_{k: k \in \mathcal{M}_{j}} x_{i j k}$. The finishing time of a machine $j_{k} \in \mathcal{M}_{j}$, denoted by $F_{j k}$, is given by

$$
F_{j k}=\sum_{i=1}^{T} x_{i j k} E T C_{i j} .
$$

Thus, the maximum finishing time of all machines (i.e., makespan), denoted by $M S(\mathbf{x})$, is given by

$$
M S(\mathbf{x})=\max _{j} \max _{k: j_{k} \in \mathcal{M}_{j}} F_{j k} .
$$

In this paper, for convenience, machines are turned off when not use, which means that the energy consumed by the bagof-tasks is given by:

$$
E(\mathbf{x})=\sum_{j=1}^{M} \sum_{i=1}^{T} x_{i j} A P C_{i j} E T C_{i j} .
$$

Let $p$ be the price customer pays and $c$ be the cost per unit of electrical energy. The profit that the organization receives by executing a bag-of-tasks is $p-c E(\mathbf{x})$. The Energy-Aware Profit Maximizing Scheduling (EAPMS) Problem defined in [1] attempting to maximize the profit per unit time can be formulated as the following nonlinear integer program (NLIP):

$$
\begin{aligned}
& \text { Maximize } \mathbf{x} \frac{p-c E(\mathbf{x})}{M S(\mathbf{x})} \\
& \text { subject to } \forall i \sum_{j=1}^{M} \sum_{k: j_{k} \in \mathcal{M}_{j}} x_{i j k}=\sum_{j=1}^{M} x_{i j}=T_{i} ; \\
& \forall j \quad F_{j k} \leq M S(\mathbf{x}), \text { for each } j_{k} \in \mathcal{M}_{j} ; \\
& \forall i, j \quad x_{i j k} \in \mathbb{Z}_{\geq 0}, \text { for each } j_{k} \in \mathcal{M}_{j} .
\end{aligned}
$$

The objective of (4) is to maximize the profit per unit time, where $\mathbf{x}$ is the primary decision variable. The first constraint ensures that all tasks of different types in the bag are assigned to some machine type. Because the objective is to maximize the profit per unit time, which is equivalent to minimize makespan, the second constrain ensures that $M S(\mathbf{x})$ is equal to the maximum finishing time of all machines.

\section{A TASK-TYPE-BASED AlgORITHM}

\section{A. Overview}

Note that (4) is a nonlinear integer program, which can not be solved optimally in polynomial time. To obtain an approximate solution of (4), one possible way is to convert (4) to an equivalent linear program (LP), and then to round the optimal fraction solution of LP to a feasible solution for (4). In [1], the authors obtained a linear program using variable substitution $r \leftarrow 1 / M S_{L B}$ and $z_{i j} \leftarrow x_{i j} / M S_{L B}$, where $M S_{L B}=\max _{j} \frac{1}{M_{j}} \sum_{i=1}^{T} x_{i j} E T C_{i j}$ is a lower bound on the makespan obtained by allowing tasks to be divided among all machines. However, the approximation of this method would be bad when the objective value is close to 0 or little tasks of type $i$ with large $E T C_{i j}$ are assigned to machines of type $j$. A similar phenomenon is also observed by Tarplee et al. [2].

To overcome the obstacle mentioned above, we will use a different method. We replace $M S(\mathbf{x})$ with a constant $M S$, and then obtain an approximate integer linear program (ILP) for (4). By rounding the optimal fraction solution for the relaxation of ILP based on the classic rounding algorithm for the generalized assignment problem [3], we obtain a feasible solution for (4). It is desired to point out that, in our method, the tasks of type $i$ such that $E T C_{i j}>M S$ will not be assigned to machines of type $j$, which is to avoid increasing the makespan too much when rounding the optimal fraction solution.

Let $L B$ be the optimal makespan by ignoring the energy consumption, and $U B$ be the makespan of the feasible schedule by assigning each task to the machine with minimum average power consumption $A P C_{i j}$. For any given constant $\epsilon>0$, Clearly, the makespan $M S\left(\mathrm{x}^{*}\right)$ of the optimal solution $\mathbf{x}^{*}$ for (4) lies in $\left[L B(1+\epsilon)^{t}, L B(1+\epsilon)^{t+1}\right]$, for some $t \in\left\{1,2, \ldots,\left\lceil\log _{(1+\epsilon)} U B / L B\right\rceil\right\}$. By trying all possible values, we will find a feasible makespan $M S$ such that $M S\left(\mathrm{x}^{*}\right) \in[M S /(1+\epsilon), M S]$, where $M S=$ $L B(1+\epsilon)^{t}$ for some $t$. For convenience, from now on, assume that $M S$ is a known constant satisfying

$$
M S\left(\mathbf{x}^{*}\right) \leq M S \leq(1+\epsilon) M S\left(\mathbf{x}^{*}\right) .
$$

For a constant $M S$, as in [1], our algorithm is decomposed into two phases. This first phase rounds the fraction optimal solution to obtain a schedule where the numbers $x_{i j}$ of tasks of type $i$ assigned to machines of type $j$ are given. The second phase assigns tasks to actual machines to produce the full task allocation $x_{i j k}$. The next two subsections describe the two phases of this recovery procedure in detail.

There are two main differences between Tarplee, Maciejewski, and Siegel's (TMS, for short) method [1] and our task-type-based (TTB, for short) method (depicted in Figure 1.): (1) The TMS method uses one fractional solution to round while we use multiple fractional solutions and choose the best one; (2) In the first phase, the energy consumption may increase in Tarplee et al.'s method while it will not increase in our method.

\section{B. b-Matching-Based Rounding}

Note that if $E T C_{i j}>M S$, the tasks of type $i$ can not be assigned to the machines of type $j$ in the optimal solution, by the definition of $M S$. This implies that $x_{i j k}=x_{i j}=$ 0 , if $i, j, k$ satisfy that $E T C_{i j}>M S$ and $j_{k} \in \mathcal{M}_{j}$. As mentioned in [1], $\frac{1}{M_{j}} \sum_{i=1}^{T} x_{i j} E T C_{i j}$ is a lower bound on $M S$. Since 


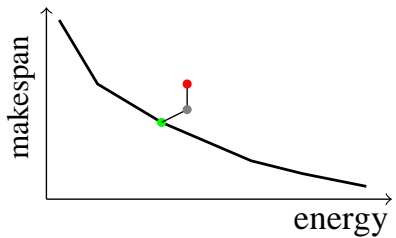

(a) TMS method

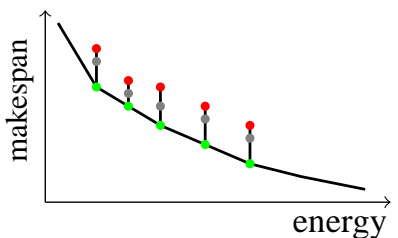

(b) TTB method
Figure 1. Comparing the main ideas of two algorithms

$M S$ is constant close to $M S\left(\mathrm{x}^{*}\right)$, we can substitute $M S$ for $M S(\mathbf{x})$ in (4). Since $p, M S, c$ are constants, the objective maximizing $(p-c E(\mathbf{x})) / M S=p / M S-c E(\mathbf{x}) / M S$ is equivalent to minimizing $E(\mathbf{x})$. Thus, we obtain an approximate equivalent integer programming formula for NLIP (4):

$$
\begin{gathered}
\text { Minimize }_{\mathbf{x}} E(\mathbf{x})=\sum_{j=1}^{M} \sum_{i=1}^{T} x_{i j} A P C_{i j} E T C_{i j} \\
\text { subject to } \forall i \sum_{j=1}^{M} x_{i j}=T_{i} ; \\
\forall j \frac{1}{M_{j}} \sum_{i=1}^{T} x_{i j} E T C_{i j} \leq M S ; \\
x_{i j} \in \mathbb{Z}_{\geq 0}, \text { for each } i, j ; \\
x_{i j}=0, \text { if } E T C_{i j}>M S .
\end{gathered}
$$

Theorem 1. Any optimal solution $\mathrm{x}^{*}$ for NLIP (4) is a feasible solution for (6).

Replacing the constraint $x_{i j} \in \mathbb{Z}_{\geq 0}$ with $x_{i j}>0$, we obtain the relaxation of (6), which is a linear program and can be solved in polynomial time. Noting that there are $T M$ variables and $T+M$ nontrivial constraints, both are less than that in the linear program (10) in [1]. By modifying Shmoys \& Tardos's rounding method in [3], which is to find a minimum-weight matching of an auxiliary bipartite graph $B(\mathbf{x})$, we can convert a feasible solution $\mathbf{x}$ for the relaxation of (6) to a feasible solution $\hat{\mathrm{x}}$ for (6). An important observation is that $\hat{\mathbf{x}}$ satisfies $M S(\hat{\mathbf{x}}) \leq 2 M S$ and $E(\hat{\mathbf{x}})=$ $E(\mathbf{x}) \leq E\left(\mathbf{x}^{*}\right)$.

Note that the running time of Shmoys \& Tardos's rounding method [3] is dependent on the number of tasks, which is very large in reality [1]. To reduce the running time, we will replace minimum-weight matching by minimumweight b-matching [4] to design an algorithm whose running time is dependent on the number of task types. For completeness, we present the modified Shmoys \& Tardos's rounding method in [3] as follows. Here, for simplicity, we only show how to construct the bipartite graph $B(\mathbf{x})$ and the edge weights, ignoring the fraction solution of the matching. Given a feasible solution $\mathrm{x}$ for the relaxation of (6), let $x_{i j}^{\prime}=x_{i j}-\left\lfloor x_{i j}\right\rfloor$, for $i=1, \ldots, T$ and $j=1, \ldots, M$.
Construct a weighted bipartite graph $B(\mathbf{x})=(U, V, E ; w)$, where $U=\left\{u_{1}, \ldots, u_{T}\right\}$ represent the set of task types. The other node set $V=\left\{v_{j s} \mid j=1, \ldots, M, s=1, \ldots, k_{j}\right\}$ consists of machine-type nodes, where $k_{j}=\left\lceil\sum_{i=1}^{T} x_{i j}^{\prime}\right\rceil$ and $k_{j}$ nodes $v_{j s}, s=1, \ldots, k_{j}$, correspond to machine type $j$, for $j=1, \ldots, M$.

As in [3], the edges in $E$ of the bipartite graph $B(\mathbf{x})$ will correspond to task-machine pairs $(i, j)$, such that $x_{i j}^{\prime}>0$. To construct the edges incident to the nodes in $V$ corresponding to machine type $j$, sort the task types in order of nonincreasing estimated times to compute $E T C_{i j}$. For simplicity, assume that

$$
E T C_{1 j} \geq E T C_{2 j} \geq \ldots \geq E T C_{T j} .
$$

If $\sum_{i=1}^{T} x_{i j}^{\prime} \leq 1$, then $k_{j}=1$, which implies that there is only one node $v_{j 1} \in V$ corresponding to machine type $j$. For each $x_{i j}^{\prime}>0$, include $\left(v_{j 1}, u_{i}\right) \in E$. Otherwise, find the minimum index $i_{1}$ such that $\sum_{i=1}^{i_{1}} x_{i j}^{\prime} \geq 1$. Let $E$ contain those edges $\left(v_{j 1}, u_{i}\right) \in E, i=1, \ldots, i_{1}$, for which $x_{i j}>0$. For each $s=2, \ldots, k_{j}-1$, find the minimum index $i_{s}$ such that $\sum_{i=1}^{i_{s}} x_{i j}^{\prime} \geq s$. Let $E$ contain those edges $\left(v_{j s}, u_{i}\right)$, $i=i_{s-1}+1, \ldots, i_{s}$, for which $x_{i j}^{\prime}>0$. If $\sum_{i=1}^{i_{s}} x_{i j}^{\prime}>s$, then also put edge $\left(v_{j, s+1}, u_{i_{s}}\right) \in E$. Finally, put edges $\left(v_{j k_{j}}, u_{i}\right) \in E, i=i_{k_{j}-1}+1, \ldots, T$, for which $x_{i j}^{\prime}>0$.

For each edge $\left(v_{j s}, u_{i}\right) \in E$, let the weight of edge $\left(v_{j s}, u_{i}\right)$ be $w\left(v_{j s}, u_{i}\right)=A P C_{i j} E T C_{i j}$. For each task-type node $u_{i} \in U$, let the capacity of $u_{i}$ be $b_{i}=\sum_{j=1}^{M} x_{i j}^{\prime}$, where $b_{i}$ is an integer as $\sum_{j=1}^{M} x_{i j}^{\prime}=\sum_{j=1}^{M} x_{i j}-\sum_{j=1}^{M}\left\lfloor x_{i j}\right\rfloor=$ $T_{i}-\sum_{j=1}^{M}\left\lfloor x_{i j}\right\rfloor$ is an integer. From the construction of the bipartite graph $B(\mathbf{x})$, it is easy to verify that there are at most $T$ nodes in $U$ and at most $\sum_{j=1}^{M} k_{j} \leq M T$ nodes in $V$. As there are $T+M$ nontrivial constraints in (6), the number of positive variables in $\mathrm{x}$ is at most $T+M$, following from the property of linear programming. Combining the fact that there are one or two corresponding edges in $E$ for each $x_{i j}^{\prime}>0$, there are at most $2(T+M)$ edges in $E$. Therefore, the minimum-cost b-matching $\mathcal{B M}$, that exactly matches $b_{i}$ times of the task-type node $u_{i}$ in $E(\mathbf{x})$, can be found by using the method in [4], whose running time is polynomial in $T$ and $M$.

The modified Shmoys \& Tardos's rounding method algorithm to construct a schedule $x_{i j}$ from a feasible solution $x$ of the relaxation of (6) is summarized as follows.

\section{Algorithm A}

Step 1. Form the bipartite graph $B(\mathbf{x})$ with weights on its edges as described above.

Step 2. Use the method in [4] to find a minimum-weight (integer) $b$-matching $\mathcal{B M}$ that exactly matches $b_{i}$ times of the task-type node $u_{i}$ in $B(\mathbf{x})$.

Step 3. For each edge $\left(v_{j s}, u_{i}\right) \in \mathcal{B M}$, assign a task of type $i$ on a machine of type $j$, which implies that $\hat{x}_{i j}=$ $\left\lfloor x_{i j}\right\rfloor+\left|\left\{\left(v_{j s}, u_{i}\right) \mid\left(v_{j s}, u_{i}\right) \in \mathcal{B M}\right\}\right|$, for every $i, j$. 
Theorem 2. [3] The schedule $\hat{\mathrm{x}}$ obtained by ALGORITHM A has makespan at most $2 M S$, and the energy consumption is at most solution $E\left(\mathrm{x}^{*}\right)$.

\section{Task-Type-Based Local Assignment}

Recall that a feasible schedule is to assign every indivisible task to a specific machine. The solution $\hat{x}_{i j}$ obtained in the last subsection is to assign $\hat{x}_{i j}$ tasks of type $i$ to machines of type $j$. To obtain a feasible schedule, we need to schedule the tasks already assigned to each machine type to specific machines within that group. In a group of machines of type $j, E T C_{i j}$ and $A P C_{i j}$ are only dependent on the task type $i$. Thus, the total energy consumed by machines of type $j$ is $\sum_{i=1}^{T} \hat{x}_{i j} A P C_{i j} E T C_{i j}$, which is a constant. Therefore, we only need to schedule tasks to minimize makespan, which is equivalent to the multiprocessor scheduling problem [5]. Tarplee et al. [1] use the common longest processing time (LPT) algorithm to assign tasks to machines for each machine type, where the $\sum_{i=1}^{T} \hat{x}_{i j}$ tasks are sorted in descending order by execution time, and each task is assigned to the machine that will complete earliest.

As shown in [1], the effect of the sub-optimality of LPT algorithm on the overall performance of the systems consider is insignificant, as the number of tasks is large generally. However, this leads to another problem, that the running time of LPT algorithm will increase dramatically when the number of tasks grows rapidly. Note that in the HPC system, the number of types of tasks is always much less than that of tasks. For example, in the simulations of [1], there are 30 task types, yet there are 11,000 tasks. An important observation is that we do not need to assign one task at a time when assign the tasks of same type.

Each group of machines of type $j$ is processed independently. The task types are sorted in descending order by execution time $E T C_{i j}$, which can be done within $O(T \log T)$ time. Without loss of generality, assume $E T C_{1 j} \geq \cdots \geq$ $E T C_{T j}$. For each machine $j_{k} \in \mathcal{M}_{j}$, let $L_{k}^{i}$ be the current load of machine $j_{k}$ after assigning tasks of type $i$, where the load of machine $j_{k}$ is the total processing time of tasks assigned to it. Initially, $L_{k}^{0}=0$ for each $j_{k} \in \mathcal{M}_{j}$. Let $A L_{i}$ be the average load of machines of type $j$ after assigning the tasks of type $i$, where

$$
A L_{i}=\frac{\sum_{k: j_{k} \in \mathcal{M}_{j}} L_{k}^{i-1}+E T C_{i j} \hat{x}_{i j}}{M_{j}} .
$$

For $k=1, \ldots, M_{j}$, assuming there are $N_{\text {unassign }}$ unassigned tasks, schedule $\min \left\{N_{\text {unassign }}, N_{k}^{i}\right\}$ tasks of type $i$ simultaneously to machine $j_{k}$, where

$$
N_{k}^{i}=\max \left\{\left\lfloor\frac{A L_{i}-L_{k}^{i-1}}{E T C_{i j}}\right\rfloor, 0\right\} .
$$

If the load of a machine $j_{k}$ is increased meaning $N_{k}^{i}>0$, we have

$$
A L_{i}-E T C_{i j}<L_{k}^{i}=L_{k}^{i-1}+N_{k}^{i} E T C_{i j} \leq A L_{i} .
$$

Obviously, there are at most $M_{j}$ unassigned tasks of type $i$, which can be assigned using LPT algorithm. It is easy to verify that our method is equivalent to the LPT algorithm in [1]. However, the running time is reduced to $O\left(\sum_{j=1}^{M}\left(T \log T+T M_{j}\right)\right)$, not depending on the number of tasks, which is always a huge number in the HPC system.

ALGORITHM B shows the pseudo-code for assigning tasks to machines for each type.

ALGORITHM B Assign tasks to machines for each type.

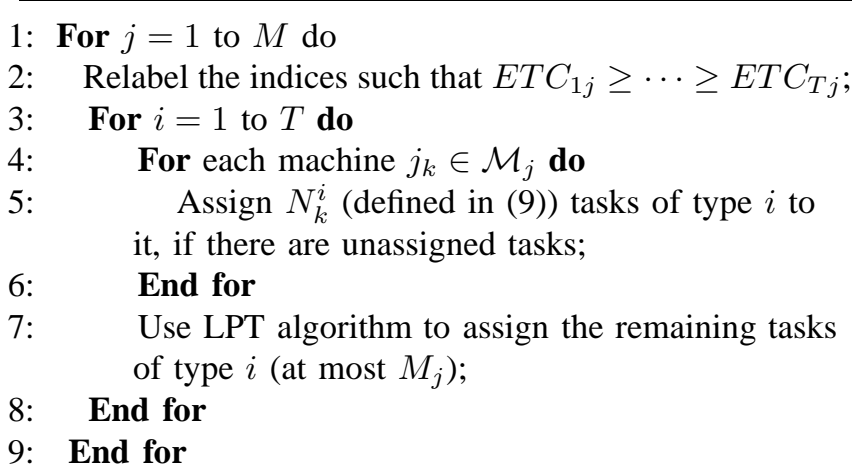

\section{Performance Analysis}

In summary, for each $t \in\left\{1, \ldots,\left\lceil\log _{(1+\epsilon)} U B / L B\right\rceil\right\}$, let $M S=L B(1+\epsilon)^{t}$. Then, use Algorithm $\mathrm{A}$ and Algorithm B to find a feasible solution for (4). Among these solutions (at most $\left\lceil\log _{(1+\epsilon)} U B / L B\right\rceil$ ), choose the one with maximum profit per unit time. It is easy to verify that the total running time is independent of the number of tasks.

For a maximization problem, if algorithm $\mathcal{A}$ can produce a feasible solution with the objective value at least $O P T / \rho$ for any instance, where $O P T$ denotes the optimal value, then $\rho$ is called the worst-case performance ratio or approximation ratio.

Combining (5) and Theorem 2, the objective of the schedule $\hat{\mathbf{x}}$ is at least

$$
\begin{aligned}
\frac{p-c E(\hat{\mathbf{x}})}{2 M S} & \geq \frac{p-c E\left(\mathbf{x}^{*}\right)}{2 M S} \geq \frac{p-c E\left(\mathbf{x}^{*}\right)}{2(1+\epsilon) M S\left(\mathbf{x}^{*}\right)} \\
& \geq \frac{1}{2+2 \epsilon} O P T .
\end{aligned}
$$

It implies that the worst-case performance ratio of the proposed algorithm is $2+2 \epsilon$, for any $\epsilon>0$.

\section{EXPERIMENTAL RESUlts}

Simulation experiments were performed to compare the quality of TMS and TTB methods. As in [1], the software was written in $\mathrm{C}++$ and the LP solver used the simplex method from COIN-OR CLP [6].

Without loss of generality, assume that $c=1$ for all the experiments. As in [1], let $E_{\min }$ be the lower bound on the 
minimum energy consumed when ignoring makespan, and $p=\gamma E_{\text {min }}$, where $\gamma=p / E_{\text {min }}$ is a parameter that will be used to affect the price per bag. Clearly, when $\gamma$ is large enough, the focus is to minimize the makespan [1]. Thus, we only consider the case that $\gamma \in[1,1.5]$.

For all the simulations, there are nine machine types and 40 machines of each type for a total of 360 machine, as in [1]. Our first experiment is based on a benchmark [7] with nine machine types and five task types, where the missing values are deleted. The workload consists of 12, 000 tasks divided among 5 task types. When $\gamma$ is varying, different solutions produced by the TMS and TTB methods are shown in Table 1 . The table shows that every solution produced by the TTB method is better than that produced by the TMS method. Especially, when $\gamma=1$, because the rounding method in the TMS method will increase the energy consumption, the TMS method produces a solution with negative objective value, while the TTB method produces the optimal solution.

\begin{tabular}{|c|c|c|c|c|c|c|}
\hline$\gamma=$ & 1 & 1.1 & 1.2 & 1.3 & 1.4 & 1.5 \\
\hline TMS & -0.6 & 985.1 & 1998.8 & 3505.4 & 5491.4 & 7933.9 \\
\hline TTB & 0.0 & 986.1 & 2009.0 & 3529.8 & 5510.7 & 7986.0 \\
\hline
\end{tabular}

Table 1. The solutions with $\gamma$ varying from 1 to 1.5

Since $E T C_{i j}$ and $A P C_{i j}$ differ slightly in the benchmark [7], to quantify the quality of the solutions in a more general case, we did 25 experiments where $E T C_{i j}$ and $A P C_{i j}$ are random numbers between 0 and 1 . In the $q$-th experiment, $q=1, \ldots, 25$, the workload consists of $150 q$ tasks divided among 30 task types. Figure 2 shows the profit per unit time computed from the TMS and TTB methods when $\gamma=1.2$. The figure shows that every solution produced by the TTB method has a higher profit per unit time. When the number of tasks is large enough, the solutions produced by two methods are close to each other.

In fact, for every experiment where $\gamma$ is also a random number we have done, the TTB method produces a higher quality solution.Moreover, in (6), letting $M S$ be the makespan of the solution produced by the TMS method, we can obtain a better solution by using the $b$-matching-based rounding and task-type-based local assignment method in Section III. It is worth to mentioning that the TTB method performs much better when $\gamma$ is small or the average number of tasks per machine is small.

\section{DISCUSSION AND FUTURE WORK}

With small modifications, our algorithm can be extended to the idle power consumption or the case where there is upper bound on the allowed power consumption, which are considered in [1]. Due to space constraint, we omit the details here.

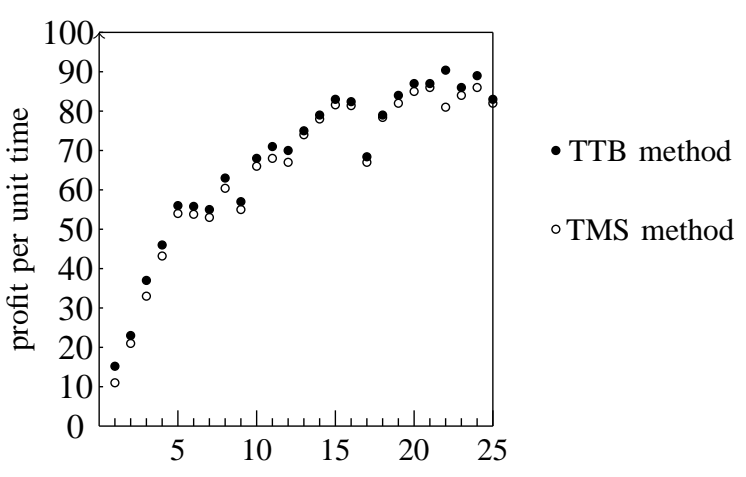

Figure 3. 25 randomized experiments

Although experiments show that the solution produced by the TTB algorithms is close to the optimal solution, this does not hold in a worst-case scenario. It is interesting and challenging to design a polynomial-time algorithm with worst-case performance ratio less than 2 .

\section{ACKNOWLEDGEMENT}

The work is supported in part by the National Natural Science Foundation of China [Nos. 11301466, 61170222], and the Natural Science Foundation of Yunnan Province of China [No. 2014FB114].

\section{REFERENCES}

[1] K.M. Tarplee, A.A. Maciejewski, and H.J. Siegel, "Energyaware profit maximizing scheduling algorithm for heterogeneous computing systems," in 14th IEEE/ACM International Symposium on Cluster, Cloud and Grid Computing, 2014, pp. 595-603.

[2] K.M. Tarplee, R. Friese, A.A. Maciejewski, and H.J. Siegel, "Efficient and scalable computation of the energy and makespan pareto front for heterogeneous computing systems," in Federated Conference on Computer Science and Information Systems, Workshop on Computational Optimization, 2013, pp. 401-408.

[3] D.B. Shmoys, and E. Tardos, "An approximation algorithm for the generalized assignment problem," Mathematical Programming 62(1-3) (1993), pp. 461-74.

[4] B. C. Huang and T. Jebara, "Fast b-matching via sufficient selection belief propagation," Journal of Machine Learning Research - Proceedings Track 15 (2011), pp. 361-369.

[5] R. Graham, "Bounds on multiprocessing timing anomalies," SIAM Journal on Applied Mathematics 17(2) (1969), pp. 416429.

[6] (2013, March) Coin-or clp. [Online]. Available: https://projects. coinor. org/Clp

[7] (2013, May) Intel core i7 3770k power consumption, thermal. [Online]. Available: http://openbenchmarking.org// result/ 1204229-SU-CPUMONITO81 